\title{
Index for Volume 14 of Molecular Plant-Microbe Interactions
}

\section{AUTHOR AND SUBJECT INDEX}

Abad, P., 72

Abbink, T. E. M., 1086

Acetobacter diazotrophicus, on sugarcane, plant growth and nitrogen effect after inoculation, 358

Actin monoubiquitylation, plant response to pathogens and symbionts, 1267

Agrobacterium spp.

-A. rhizogenes: A4-orf8 gene, biological role, 405; on Medicago truncatula, root transformation, nitrogen fixation, endomycorrhizae, 695

-A. tumefaciens: C58 chromosome, gene integration, 577; gall inhibition by mannopine and opine, 793; tetracycline resistance mutation, 98; transformable vector, in Lotus japonicus, chromosome library, 422

Aguilar, O. M., 55

Ahmad, M., 1213, 1223

Ahn, I.-P., 496, 1340

Ahn, J.-H., 1436

Akimitsu, K., 749

Alfalfa

—root nodules, $n g l 9$ gene, 1463

-Sinorhizobium meliloti: ilvC mutants, 55; on, symbiosis and oxidative burst, 86

- superoxide dismutases, in nodules and leaves, isozyme activities, 1178

Alfalfa mosaic virus

-movement and coat proteins, virus transport role, 1051

-movement protein mutants, localization and phenotypes, 1063

Ali, S., 675

Allen, R., 536, 1247

Alternaria spp.

- A. alternata: on citrus, endopolygalacturonase relation, 749 ; on tomato, tobacco resistant to, 460

$-A$. citri, on citrus, endopolygalacturonase, 749

Altier, D. J., 947

Alvarez-Morales, A., 545

Andreotti, S., 255

Apple, scab resistance, receptor-like genes, 508

Arabidopsis

- calmodulin-like domain protein, oxidase activity and oxidative burst in tomato protoplasts, 1261

- downy mildew, resistance gene and salicylic acid, 416

- protoplast, tubules from DNA construct, Cauliflower mosaic virus, cover photo, August

Arabidopsis spp.: NIM1 gene, effect on resistance and fungicide effectiveness,
1114; Pseudomonas syringae and

Peronospora parasitica on, resistance, $R$ gene pathway, EDS1-mediated, 1131

-A. thaliana: Azorhizobium caulinodans on, in xylem, 93; colonization by

Rhodococcus fascians, 599; downy

mildew, callose response and

hypersusceptibility, salicylate

hydroxylase, 439; ethylene signal

transduction, nematode susceptibility,

206; Peronospora parasitica on, induction by salicylic acid and NIM1/NPR1independent genes, 1235; Pseudomonas syringae, hypersensitivity and $h r p$ genes, 234; root-knot nematode on, compatible interactions and genes, 288

Archer, D., 367

Arnould, C., 737

Arrese-Igor, C., 1178

Arroyo-Rodriguez, A. S., 1213

Aspergillus spp.

-A. flavus: on maize, growth inhibition by protein from Lablab purpureus, 955; on maize, lipoxygenase response, 980

- A. giganteus, protein from, inhibitor of fungal pathogens, 1327

-A. nidulans, A. flavus compared to, maize ribosome-inactivating protein, 164

Avocado, anthracnose, $p e l \mathrm{~B}$ as virulence factor, 988

Axtell, M. J., 181

Ayaydin, F., 609

Azorhizobium caulinodans

—on Arabidopsis, xylem colonization, 93

-invasive behavior on host, cover photo, July

-on Sesbania rostrata, symbiosis disablement, synthase, 857

Azospirillum brasilense, membrane protein purification, root affinity, 555

Bacteria

- Gram-negative, GacS/GacA twocomponent system, regulatory roles, 1351

-hydrocarbon-degrading, rhizostimulation, plant-bacterium pair as tool, 1197

Bae, C.-Y., 80

Baek, C.-H., 793

Bailey, A., 1303

Balconi, C., 1255

Balestrini, R., 255

Ballvora, A., 629

Banba, M., 173

Barash, I., 431

Barker, D. G., 693, 737

Barley, Pyrenophora teres, pathogenicity, kinase gene for activation, 116

Barta, F., 1395

\section{ERRATA PAGE NUMBERS IN ITALICS}

Bassüner, R., 42

Batut, J., 887

Bauer, W. D., 775

Baum, T. J., 536, 1206, 1247

Beachy, R. N., 895

Becana, M., 1178, 1189

Bécard, G., 693

Becker, D., 832

Begum, A. A., 1404

Bennett, M., 267

Beno-Moualem, D., 988

Beynon, J. L., 416

Bianciotto, V., 255

Bindslev, L., 1368

Biological control

-Pseudomonas chlororaphis: for Fusarium oxysporum on tomato, 969 ; for tomato foot and root rot, $p h z H$ gene role, 1006 - putrescine in rhizosphere, bacterial inhibition, 1096

Bipolaris maydis, maize resistance, protein and global mRNA profile, 947

Bird, D., 1168

Bittner-Eddy, P. D., 416

Blackburn, M., 905

Blissett, K., 367

Bloemberg, G. V., 969, 1006, 1096, 1197

Blume, B., 867

Blumeria graminis, PKA-c gene, complementation with Magnaporthe grisea, $\triangle$ cpkA mutation, 1368

Boesten, B., 471

Boisson-Cernier, A., 693

Bol, J. F., 1051, 1063, 1086

Bolaños, L., 663

Bonas, U., 629

Bonfante, P., 255

Bonilla, I., 663

Borgstrøm, B., 707

Borisov, A. Y., 471

Borzi, J., 887

Boston, R. S., 164

Botrytis cinerea

-infection process, $\mathrm{G}$ protein alpha subunit role, 1293

-resveratrol and fenpiclonil sensitivity, 562

Boulton, M. I., 609

Bové, J. M., 225

Bowyer, P., 1303

Boyko, V., 895

Bradshaw, N., 367

Bradyrhizobium japonicum

— pattern in cowpea nodules, 1286

-on soybean, genistein sensitivity, altered nod gene regulation, 1404

Brandwagt, B. F., 460

Bras, C. P., 839

Braun, E., 1376

Publication no. M-2001-1107-02O 
Brown, I. R., 394

Burdman, S., 555

Burger, A., 1312

Buris, R. H., 358

Cabral, S., 1274

Cade, R., 1114

Calamante, G., 1274

Candidatus, phytoplasma on Catharanthus roseus, gene regulation, 225

Candresse, T., 278, 804, 1032

Caprari, C., 1436

Capsicum annиum, appressorium formation, inhibition by esterase, 80

Caputo, E., 250

Carmen, R.-R., 116

Carrot, mycorrhizal roots, biofilm and biocontrol, 255

Caspi, M., 1463

Castagnaro, A. P., 925

Castagnone-Sereno, P., 72

Catharanthus roseus, mollicute infections, gene regulation, 225

Cauliflower mosaic virus

-hypersensitivity in Nicotiana, resistance coupled with cell death, 31

- on Nicotiana, cover photo, January

- protein fusion and virus movement tubule formation, 1026

Cebrián, A., 663

Chabaud, M., 693

Chang, J. H., 451

Charles, T. C., 1404

Chatterjee, A., 516

Chatterjee, A. K., 516

Chaufaux, J., 214

Chechetka, S. A., 848

Cheern, M., 204

Cheon, C.-I., 1286

Chin-A-Woeng, T. F. C., 969, 1006

Chittoor, J. M., 1411

Chun, J.-Y., 1286

Ciardi, J. A., 487

Cinege, G., 887

Ciuffetti, L. M., 675

Cladosporium fulvum

-alcohol oxidase pathogenicity factor, aldehyde dehydrogenase dispensability, 367

—on tomato, avirulence gene Avr9, nitrogen response factor, 316

- on tomato, $C f-9$ resistance gene, dilysine motif, 412

- on tomato, gene cluster and resistance, 508

Clarke, J. D., 1131

Clavibacter michiganensis

- cytoplasmic membrane, fragarin effect, 925

- on tomato, transposon mutagenesis system, 1312

Clayton, K., 42

Clemente, T. E., 98

Cochliobolus carbonum, $\beta$-glucanase genes, mutational analysis, 1436

Cocking, E. C., 93

Colas, V., 326

Cole, A. B., 31

Colletotrichum spp., pH modulation, virulence mechanism, 1105
-C. gloeosporioides: on avocado, pelB as virulence factor, 988; on pepper, pepper esterase gene, inhibition, 80

- C. laginarium, on cucumber, growth conidiation, and appressorium formation, kinase role, 1149

Collmer, A., 386

Colon, L. T., 996

Conrod, S., 326

Conway, W. S., 1105

Coplin, D. L., 431, 1213, 1223

Corn (see also Maize): Stewart's wilt, harpin biological activity, 1223

Cornelis, K., 599

Corran, A., 1303

Cover photo

-Arabidopsis protoplast, tubules from transfected DNA construct, August

-Arabidopsis spp., Peronospora parasitica, NIM1 gene display, September

-Azorhizobium caulinodans, invasive behavior of host, July

- Cauliflower mosaic virus on Nicotiana, January

- Glomus intraradices, mycelium, February

-Medicago truncatula, gene transcription, nodulation stages, June

-Peronospora parasitica on Arabidopsis, laser scanning confocal microscope, April

-Phaseolus vulgaris, actin and ubiquitin colocalization in root nodule cells, confocal microscope, November

-Puccinia coronata, Avena Rds loci mediation, hypersensitive cell death, December

-Rice, blast, sheath blight, transgenic plants, October

-Soybean mosaic virus, N graft inoculation, hypersensitivity, May

- Tobacco, leaf endoplasmic reticulum retrieval-retention signal, agroinfiltration, March

Cowpea, nodules, unique development pattern, 1286

Crasta, O., 947

Crespi, D., 55

Csanádi, G., 887

Cucumber

- anthracnose, growth, conidiation, and appressorium function, kinase role, 1149

- Hop stunt viroid, ribonucleoprotein complex, 910

Cucumber mosaic virus

-DNA infection system, plasmodesmata, 126

- protein sequences, infectivity and movement, 378

- transgene RNA silencing, salicylic acidmediated resistance, 715

Cui, Y., 516

Cullimore, J. V., 267

Cyclophilin, yellow lupin, accumulation in nodule meristem, 1384

Daboussi, M.-J., 308

Dalton, D. A., 1189

Danet, J.-L., 225

Daniels, M. J., 768, 785

Dantán-González, E., 1267

D'Aoust, F., 1404
Davey, M. R., 93

Davies, J. W., 196, 609

Davis, E. L., 63, 536, 1247

de Billy, F., 267, 737

de Boer, J. M., 536

Dedieu, A., 737

Défago, G., 639

Delaney, T. P., 439, 1235

De Lorenzo, G., 867

del Pozo, A. M., 1327

Del Sorbo, G., 562

de O. Manes, C.-L., 189

De Rycke, R., 857

de Voer, G., 969

de Vogel, J., 1086

De Waard, M. A., 562

Dewbre, G. R., 1140

De Wit, P. J. G. M., 316, 412, 867

D'Haeze, W., 857

Dhandaydham, M., 1168

Dharmapuri, S., 1335

Diaz-Ricci, J. C., 925

Diederichsen, E., 1075

Dietrich, R., 1114

Ding, B., 905, 1332

Ding, S.-W., 715

Ding, X., 536

Dinoor, A., 988

Di Pietro, A., 653

Doke, N., 725

Dombrecht, B., 426, 918

Dong, X., 1131

Donofrio, N. M., 439, 1235

Dow, J. M., 768, 785

Dowd, P. F., 947

Driscoll, B. T., 1404

Dudits, D., 609

Dufresne, M., 300

Dulguerova, G., 555

Dunn, K., 1463

Dusha, I., 887

Eichenlaub, R., 1312

El-Gachtouli, N., 737

Elicitor, harpin, Erwinia carotovora, role in two-component system, 516

Epel, B. L., 895

Erratum, vol. 14, no. 3, 2001, 1032

Erwinia spp.

-E. carotovora: $h r p$ gene cluster, role in virulence, 962; RsmA-rsmB system, extracellular enzyme gene expression, 931; on transgenic plants, pheromone enhances resistance, 1035; twocomponent system, enzymes and harpin, 516

-E. chrysanthemi: mutations for virulence, 386; negative regulator ExuR, galacturonate transport, 816; pathogenicity and superoxidase dismutase, 758; virulence factors, nucleoidassociated protein role, 10

-E. herbicola, gene cluster and operon, genetic organization, 431

Erysiphe graminis, on wheat, resistance enhanced by genes, 832

Expert, D., 758

Faelen, M., 10

Fakhoury, A. M., 955 
Farías, R. N., 925

Farrall, L., 671

Farrand, S. K., 98

Ferraioli, S., 250

Filippone, M. P., 925

Flis, B., 1420

Folimonov, A., 1332

Folkerts, O., 947

Fontanet, P., 1327

Forster, R. L. S., 939

Foxworthy, A., 775

Fraaije, B. A., 156

Francl, L. J., 675

Franza, T., 758

Frederick, R. D., 1213

Friedrich, L., 1114

Frugier, F., 55

Frugoli, J., 1364

Fry, J., 1016

Fujita, Y., 580

Fungicides

- effectiveness enhanced by NIM1 gene, 1114

-fenpiclonil, Botrytis cinerea sensitive to, 562

Fusarium spp.

-F. moniliforme, Aspergillus giganteus protein, inhibition, 1327

$-F$. oxysporum: on melon, pathogenicity and arginine biosynthesis gene, 580; subtilase from, molecular characterization, 653; on tomato, control by Pseudomonas chlororaphis, phenazine1-carboxamide role, 969; on tomato, foot and root rot control by Pseudomonas, phzH gene, 1006

$-F$. verticillioides, on maize, lipoxygenase response, 980

Gaeumannomyces graminis, tissue-specific requirement, definition, 300

Ganal, M., 629

Gao, B., 1247

Gao, M., 775, 857

Garcia, F., 693

García, J. A., 278, 1032

García-Olmedo, F., 386

Gardner, H. W., 980

Gardner, R. C., 939

Garnier, M., 225

Gartemann, K.-H., 1312

Gebhardt, C., 1420

Geiger, O., 349

Gelvin, S. B., 577

Gene

-A4-orf8, in Agrobacterium rhizogenes, biological activity, 405

- AUX1, role in Medicago truncatula, 267

-Avr9, in Cladosporium fulvum, 316

- $\beta$-1,4-endoglucanase, cyst nematode on soybean, 63

- $\beta$-glucanase, Cochliobolus carbonum, 1436

-C2, Tomato yellow curl virus, pathogenicity determinant, 1125

- Cf-9, tomatoes resistant to Cladosporium fulvum, 412

- Cf9 and Avr9, Leptosphaeria maculans on Brassica napus, resistance, 1075

-in Cladosporium fulvum, 316
-Erwinia chrysanthemi, nucleoidassociated protein role, virulence, 10

- flhF, Xanthomonas oryzae, flagellar operon region, 204

-hrp, Xanthomonas campestris on pepper, 785

- hrp cluster, Erwinia carotovora and role in virulence, 962

-kinase, mitogen activated, barley net blotch, 116

-LeETR4, tomato, hypersensitivity and Xanthomonas, 487

$-m y b$, role in rice blast, 527

— ngl9, alfalfa root nodules, 1463

-nod, Bradyrhizobium japonicum on soybean, genistein sensitivity, 1404

-ntrR, mutation, Sinorhizobium meliloti, 887

-ORP1, Magnaporthe grisea on rice, 308

-pelB, Colletotrichum gloeosporioides on avocado, virulence factor, 988

-PepEST, on pepper, appressorium formation inhibition, 80

-phytoplasma protein, 1043

-Pti5, tomato, defense gene to Pseudomonas syringae, 1453

-Pto, potato speciation, ancient diversification, 996

-rice-Magnaporthe grisea interaction, analysis, 1340

- RPS2 and avrRpt2, in Pseudomonas syringae, 181

- Rsv1, Soybean mosaic virus, hypersensitive response, 587

-in transgenic wheat, resistance enhanced by, 832

Gessler, C., 508

Gheysen, G., 288

Gianfranceschi, L., 508

Gianinazzi-Pearson, V., 737

Ginseng, Nectria radicicola, RNA regulation, 496

Giroux, M. J., 1255

Glomus intraradices

-mycelium, cover photo, February

- phosphate transporter gene, regulation, 1140

Goellner, M., 536

Goethals, K., 189, 599

Gómez, G., 910

Gong, F.-C., 775

González, E., 1178

Goodman, R. M., 1458

Goosen, T., 316

Gorfer, M., 135

Görlach, J. M., 1436

Govers, F., 996, 1444

Grant, S., 947

Grasso, D. H., 55

Greenberg, J. T., 145

Gresshoff, P. M., 422

Grgurina, I., 336, 1426

Grønlund, M., 839

Gronover, C. S., 1293

Grosgeam-Cournoyer, M.-C., 308

Grosjean, C., 267

Gross, D. C., 336, 1426

Guikema, J. A., 1411

Guilhabert, M. R., 701

Gunapala, N., 358
Guolter, K., 225

Gutierrez-Corona, J. F., 653

Guttman, D. S., 145

Györgypál, Z., 887

Haas, D., 1351

Hajimorad, M. R., 587

Hamer, J. E., 877

Han, Y., 1026

Hanif, M., 135

Harpin, Pantoea stewartii, biological activity, 1223

Harrison, M. J., 1140

Hart, P., 234

Hata, S., 173, 477

He, P., 1453

He, S. Y., 234

Heeb, S., 1351

Heinlein, M., 895

Helfer, A., 405

Hennin, C., 1075

Hernádez-Guzmán, G., 545

Hérouart, D., 86

Heterodera spp.

$-H$. glycines, on soybean: cDNA peptide selection, 536; gene mapping, 42; parasitism genes in esophageal glands, 1247

-H. schachtii, on Arabidopsis thaliana, ethylene signal transduction role, 1206

Hilaire, E., 1411

Hill, J. H., 587

Hille, J., 460

Hiremath, S. T., 618

Hodges, T. K., 877

Hoffman, L. M., 701

Hoffmann, K., 242

Höfte, M., 1075

Hollomon, D. W., 156

Holsters, M., 189, 599, 857

Holzer, F. M., 214

Hong, Y., 1125

Hop stunt viroid

- phloem lectin involvement, long-distance, 905

- viroid RNA and phloem protein relation, ribonucleoprotein complex, 910

Horváth, G. V., 609

Howell, S. H., 1026

Hoyos, M. E., 1223

Hsu, C. S., 181

Hu, W., 234

Huang, M., 1063

Huang, Z., 1026

Hua-Van, A., 308

Huertas-González, M. D., 653

Hugouvieux-Cotte-Pattat, N., 10

Humara, J. M., 577

Hussey, R. S., 72, 536, 1247

Hutchison, M. L., 336

Hypersensitivity

-Arabidopsis in tomato, hrp genes, 234

-Cauliflower mosaic virus on Nicotiana, 31

- oat crown rust, gene-for-gene resistance, $R d s$ and Rih, 1376

-Potato virus $X$ on transgenic tobacco, resistance and symptoms, 1274

- Pseudomonas syringae on bean, 90

- Soybean mosaic virus, Rsv1-mediated resistance, 587 
-Tobacco mosaic virus: chimeric helicase sequences, 1086; on tobacco leaves, jasmonic acid accumulation, 261

Hyun Ryu, K., 378

Hyytiäinen, H., 931

Iaccarino, M., 823

Ikuta, A., 848

Imaizumi-Anraku, H., 848

Infection, tissue-specific requirements, by

Magnaporthe grisea and

Gaeumannomyces graminis, 300

Inoue, I., 580

Ip, H., 1404

Ishizaka, M., 848

Isshiki, A., 749

Itaya, A., 1332

Iturbe-Ormaetxe, I., 1189

Izui, K., 173

Jagoueix-Eveillard, S., 225

Jakobek, J. L., 90

Jaziri, M., 599

Ji, L.-H., 715

Jin, Q.-L., 234

Jockusch, H., 914

Johansen, I. E., 707

Jones, A. R., 775

Jones, J. B., 487

Jones, J. D. G., 867

Jongejan, L., 1063

Joosten, M. H. A. J., 316, 412, 867

Journet, E.-P., 737

Jung, H., 1043

Jurkevitch, E., 555

Kakizawa, S., 1043

Kamoun, S., 996

Kang, S., 671

Kassem, M. A., 422

Kasulke, D., 1293

Katz, A., 895

Kawaguchi, M., 848

Kawakita, K., 725

Keen, N. T., 988

Keller, H., 326

Keller, N. P., 980

Kennedy, C., 358

Kereszt, A., 1395

Kershaw, M. J., 1368

Kiba, A., 111

Kijne, J. W., 839

Kim, H., 1436

Kim, K.-S., 793

Kim, S. (Korea), 1340

Kim, S. J. (Mich.), 618

Kim, Y. S., 80

Kinase, Colletotrichum lagenarium, role in growth, conidiation, and appressorium formation, 1149

Király, L., 31

Kirchner, O., 1312

Kirkpatrick, B. C., 701

Kiss, E., 887, 1395

Klee, H. J., 487

Knee, E. M., 775

Kneppers, T. J. A., 460

Ko, M. K., 80

Kobayashi, K., 1274

Koiv, V., 1035
Kojima, K., 1149

Koltai, H., 1168

Komeda, K., 1149

Kondorosi, Á., 572, 887, 1395

Kotlizky, G., 895

Kouchi, H., 173, 848

Krause-Sakate, R., 804

Krishnamurthy, K., 1255

Kuboyama, T., 1043

Kuiper, I., 1096, 1197

Lablab purpureus, lectin-like protein from, Aspergillus growth effect, 955

Lacadena, V., 1327

Laccaria bicolor, symbiosis-regulated ras, characterization, 618

Lahaye, T., 629

Laitiainen, E., 135

Lamberti, A., 250, 823

Lapidot, M., 895

Laporte, M.-L., 758

Lawton, K., 1114

Leach, J. E., 1411

Lebrun, M.-H., 308, 671

Lee, H. H., 80

Lee, J. K., 793

Lee, L.-Y., 577

Lee, M.-L., 1286

Lee, M.-W., 527, 685

Lee, Y.-H., 80, 496, 1340

Le Gall, O., 804

Legumes

-models, meeting report in Germany, 6

—nodule mitochondria, antioxidants of, 1189

Leptosphaeria maculans, on Brassica napus, resistance enhanced by $C f 9$ and $A v r 9$ genes, 1075

Lettuce mosaic virus, pathogenicity determinants, viral genome map, 804

Leverentz, B., 1105

Lewers, K., 42

Li, Q., 378

Li, X., 1131

Lightfoot, D., 422

Lindgren, P. B., 90

Linthorst, H. J. M., 1086

Liu, H., 1125

Llama-Palacios, A., 386

Loh, Y.-L., 816

Lohar, D., 422

Lomonossoff, G. P., 196

López, J. C., 55

Lopez, O., 572

López-Lara, I. M., 349

López-Solanilla, E., 386

Lörz, H., 832

Lot, H., 804

Lotus japonicus

-model for genomic analysis of Rhizobium, 422

—nodulation, root cortical cell effect, 839

-nodulation factors, Mesorhizobium loti, 848

Lu, S.-E., 1426

Luderer, R., 867

Lugtenberg, B. J. J., 969, 1006, 1096, 1197

Luo, Z.-Q., 98

Luyten, E., 678, 918

MacDiarmid, R. M., 939

Mackenzie, S., 42
Mäe, A., 1035

Maeda, H., 725

Magnaporthe grisea

-avirulence gene, Pot 3 transposon insertion, virulence gain, 671

- complementation of $\triangle c p k A$ mutation and Blumeria graminis, PKA-c gene, 1368

-inhibition by Aspergillus giganteus, protein, 1327

—on rice: gene analysis, 1340; gene tagging using transposon impala, 308; pathogenesis-related gene family, 877; tissue-specific requirements, definition, 300

Maier, F. J., 116

Maier, T., 536, 1247

Maize (see also Corn)

—ear rot, Aspergillus inhibition by protein from Lablab purpureus, 955

- oxygenase response, seed-infesting fungi, 980

-ribosome-inactivating protein, effect on Aspergillus spp., 164

- southern corn leaf blight, resistance and proteins and global mRNA profiles, 947

Maize streak virus, phase-specific activation promoters, cell-cycle, 609

Majerczak, D. R., 1213, 1223

Maldonado, S., 1274

Maldonado-Mendoza, I. E., 1140

Malik, K., 1261

Mandrich, L., 823

Mansfield, J. W., 394

Manulis, S., 431, 1213

Marczewski, W., 1420

Martens, A., 996

Martinez, J. P., 675

Martínez-Cadena, G., 653

Matamoros, M. A., 1189

Matsuda, Y., 1332

Matsunaga, M., 580

Mattei, B., 867

May, S., 267

Mayama, S., 477

McEvoy, J. L., 1105

McGee, J. D., 877, 1411

McNellis, T. W., 181

Mcquattie, C. J., 618

Medicago truncatula

- Agrobacterium rhizogenes-transformed roots, nitrogen fixation and symbiotic association, 695

- auxin and Auxl-like genes, nodulation, 267

—early nodulin gene, mycorrhization, 737

-model legume, genetic nomenclature guidelines, 1364

-nodulation stages, cover photo, June

Méglecz, E., 653

Meksem, K., 422

Meloidogyne spp., on Medicago truncatula, relation to Sinorhizobium meliloti, 1168

-M. incognita: on Arabidopsis thaliana, compatible genes, 288; avirulence protein, molecular cloning, 72

Men, A. E., 422

Mendgen, K. W., 1319

Mentaberry, A., 1274

Mersch, B., 914

Meshi, T., 126, 1158 
Mesorhizobium loti, on Lotus japonicus, nodulation factor, 848

Michael, A. J., 1303

Michelmore, R. W., 451

Michiels, J., 426

Miki, B. L., 1261

Mills, D. A., 701

Min, K.-H., 1286

Minchin, F. R., 1178

Miranda, J., 572

Miyata, S., 1043

Moënne-Loccoz, Y., 639

Mollicute, on Catharanthus roseus, gene regulation, 225

Montagu, M. V., 189

Montesano, M., 931, 1035

Moore, C. J., 939

Mor, H., 431

Morandi, D., 737

Moreara, C., 572

Mort, A. J., 775

Moyer, J. W., 242

Mucilage, pea root, rhizosphere bacteria, 775

Mudgett, M. B., 181

Mueller, E., 1303

Mycorrhizae

- Agrobacterium rhizogenes on Medicago truncatula, symbiosis, 695

- Laccaria bicolor, symbiosis-regulated ras, 618

-Medicago truncatula, early nodulin gene, 737

Mycosphaerella graminicola, on wheat, carbohydrate metabolism, transformance and proteins, 156

Mycotoxin (see Toxin)

Myovirus, on Nectria radicicole, virulence regulation, 496

Nakayashiki, H., 477

Namba, S., 1043

Namiki, F., 580

Nasser, W., 10

Nectria radicicola, virulence regulation, viral dsRNA role, 496

Nelson, R. S., 1332

Nennstiel, D., 867

Newman, M.-A., 785

Nicotiana spp., (see also Tobacco): toxin sensitivity, tomato colonization, 460

- N. benthamiana: Pea early browning virus, replicase-derived resistance, 196; Potato virus $X$, antimicrobial defensin, vector, 111

-N. edwardsonii, Cauliflower mosaic virus, resistance and cell death uncoupling, 31

$-N$. tabacum, colonization by Rhodococcus fascians, 599

Nielsen, K., 164

Nijkamp, H. J. J., 460

Nijsse, J., 599

Nikovics, K., 609

Nishi, K., 580

Nishigawa, H., 1043

Nishihara, M., 111

Niwa, S., 848

Nizan, R., 431

Nodulation

-AUX1-like genes in Medicago truncatula, 267 -competitiveness, Rhizobium

leguminosarum, myo-inositol catabolism, 1016

-Mesorhizobium loti on Lotus japonicus, lipochitin factor, 848

-on pea: boron deficiency in nodules, 663; gene mutations, 471

- Phaseolus vulgaris, actins, monoubiquitylation, 1267

- protein NodG, in Rhizobium leguminosarum, 349

- Rhizobium etli on: Lotus japonicus, early senescence, 173; Phaseolus vulgaris, arginine biosynthesis, 250

-root cortical cell effect, 839

Nomenclature, genetic, guidelines, model legume Medicago truncatula, 1364

Noreen, S., 1096

Novacky, A., 1223

Nuc, K., 1384

Nuc, P., 1384

Nürnberger, T., 867

Oat, infection and elicitors, apoptotic response, 477

O'Callaghan, K. J., 93

Oh, B.-J., 80

Oh, H.-S., 1286

Ohashi, Y., 261

Okon, Y., 555

Okuda, M., 580

Okuno, T., 1149

Oláh, B., 887

Oldach, K. H., 832

Olivares, J., 811

Oliver, R. P., 367, 1368

Opperman, C., 1168

Osbourn, A. E., 300

Oshima, K., 1043

Otten, L., 405

Ottum, S. A., 675

Ownes, R. A., 905

Pallás, V., 910

Palukaitis, P., 378

Palva, E. T., 962, 1035

Panabières, F., 326

Panicum mosaic virus, capsid protein of satellite, multiple biological roles, 21

Pantoea stewartii

- gene cluster and sequence analysis, genetic organization, 1213

-harpin produced by, biological activity, 1223

Pardo, A. G., 135

Park, H.-J., 725

Parr, A., 785

Parrand, S. K., 793

Pasternak, T., 609

Patocchi, A., 508

Patriarca, E. J., 250, 823

Pautot, V., 214

Payne, A. C., 156

Payne, G. A., 164

Pea

-root mucilage, rhizosphere bacteria and carbon source, 775

-root nodulation, boron deficiency, glycoprotein, 663

Pea early browning virus, on Nicotiana benthamiana, replicase-derived resistance, 196

Pea seedborne mosaic virus, mutation and protein VPg, pathotype-specific virulence, 707

Peres, A., 609

Pérez-García, A., 316

Peronospora parasitica

—on Arabidopsis: laser scanning confocal microscope, cover photo, April; NIMI gene display, cover photo, September; resistance gene and salicylic acid, 416; resistance and $R$-gene pathway, 1131 ; salicylic acid, NIM1/NPR1-independent gene induction, 1235

- on Arabidopsis thaliana, callose response and hypersusceptibility, salicylate hydroxylase, 439

Perotto, S., 255

Phaseolus vulgaris

- actin and ubiquitin colocalization in root nodule cells, confocal microscope, cover photo, November

- actins in root nodules, transient modification, 1267

—nodulation, root cortical cell effect, 839

-nodulation and arginine biosynthesis, essential gene, 250

—nodules, antioxidants in mitochondria, 1189

Pheromone, bacterial, Erwinia carotovora on transgenic plants, 1035

Phosphate, regulation by phosphate transporter gene, in Glomus intraradices, 1140

Phytoalexin

- phytoanticipins comparison, Clavibacter michiganensis, 925

-resveratrol, Botrytis cinerea sensitive to, 562

Phytophthora spp.

$-P$. infestans: Aspergillus giganteus protein, inhibition, 1327; on potato, arachidonic acid and salicylic acid relation, 725 ; on potato, chromosomal deletions and virulence on R3, R10, R11 lines, 1444

$-P$. parasitica, on tobacco, elicitin genes, 326

Phytoplasma, protein translocation genes, cloning and analysis, 1043

Phytotoxin

- coronatine, Pseudomonas syringae on tomato, wound response, 214

- syringomycin and syringopeptin, gene clusters, localized in 145-kb DNA region, map, 1426

Pichon, M., 737

Pierre, M., 629

Pike, S., 1223

Pines, O., 988

Pisum sativum, gene mutation, nodule development effect, 471

Plum pox virus, pathogenicity determinant, 278, 1032

Podila, G. K., 618

Poinsot, V., 678

Poole, P. S., 1016

Potato (see also Solanum spp.)

-late blight, chromosomal deletions in isolates, virulence, 1444 
- Phytophthora infestans, arachidonic acid and salicylic acid, 725

Potato leafroll virus, resistance trait locus, chromosome XI, $N$-gene-like marker, 1420

Potato spindle tuber viroid, on tomato, RNA silencing, 1332

Potato virus $X$

- cell-to-cell movement, p12 and p8 roles, 1158

-on transgenic tobacco, resistance and symptoms, 1274

- vector, in tobacco, defensin production, 111

Potato virus $Y$, on transgenic tobacco, specific resistance and ring symptoms, 1274

Priefer, U. B., 471

Prinsen, E., 189

Promé, J.-C., 678

Prusky, D., 988, 1105

Pseudomonas spp., polyketide synthase gene phlD, polymorphism, 639

-P. chlororaphis: biocontrol agent for tomato foot and root rot, $p h z H$ gene role, 1006; tomato foot and root rot controlled by, phenazine-1-carboxamide, 969

$-P$. fluorescens: on carrot, biocontrol strain mutants, mycorrhizae, 255; root colonization, putrescine in rhizosphere, 1096

-P. syringae: on Arabidopsis, resistance and avirulence genes, 181; on Arabidopsis, resistance and $R$-gene pathway, 1131; avirulence protein, mutational analysis, 451; on bean, amidinotransferase gene coding, phaseolotoxin, 545; on bean, cDNA clone, hypersensitivity, 90; genomic integration system, effectors AvrRpt2 and AvrRpml, 145; syringomycin and syringopeptin gene clusters, 145-kb DNA region, map, 1426; syringopeptin and syringomycin role, biosynthesis mutant analysis, 336; on tomato, defense genes, Pti5

overexpression, 1453; on tomato, effector protein transfer, Hrp pilus role, 394; on tomato, hypersensitivity and hrp genes, 234; on tomato, wound response, coronatine, 214

Puccinia spp.

-P. coronata, Avena Rds loci mediation, hypersensitive cell death, cover photo, December; gene-for-gene resistance, hypersensitivity, 1376

$-P$. recondita, on wheat, resistance enhanced by genes, 832

Puppo, A., 86

Putnoky, P., 1395

Pyrenophora spp.

$-P$. teres, on barley, pathogenicity and kinase gene, 116

$-P$. tritici-repentis, ToxB gene, characterization, 675

Pyricularia grisea, on rice

-defense-related genes, identification, 685

- jasmonic acid-inducible gene, infection and cell death, 527

Qi, M., 527, 685

Qiu, W. P., 21, 242
Quinto, C., 1267

Quiot, J.-B., 278, 1032

Quiot, L., 278, 1032

Rairdan, G. J., 1235

Rajes, D., 914

Ramette, A., 639

Ramos, J., 1178

Rantakari, A., 962

Raudaskoski, M., 135

Redondo, E., 804

Redondo-Nieto, M., 663

Resistance

- cell death and hypersensitivity,

Cauliflower mosaic virus on Nicotiana, 31

- Cucumber mosaic virus, salicylic acid mediation, 715

-Lettuce mosaic virus, pathogenicity determinants, 804

-rice to fungi, wheat puroindoline role, 1255

- tobacco to Tobacco mosaic virus, salicylic acid effect, 1458

- tomato and tobacco, Tomato spotted wilt virus, maps, 242

Reuhs, B. L., 1395

Reverchon, S., 10

Reviewers, acknowledgement, 3

Rhizobium sp.: cytochrome homolog, symbiosis specificity, analog, 918; lipochitooligosaccharide molecules, complex mixture, 678; RK2-derived cloning vector, gene analysis, 426

-R. etli: on Lotus japonicus, early root nodules, 173; on Phaseolus vulgaris, gene $\arg C$ essential for nodulation, 250

$-R$. leguminosarum: on bean, antioxidants in nodule mitochondria, $1189 ; \mathrm{NH}_{4}$ assimilation capacity, Gstl protein, 823; nodulation competitiveness, myo-inositol catabolism, 1016; nodulation protein, protein reductase carrier, 349; symbiotic genes, mutation effect, 471

Rhizosphere

-bacteria, on pea root mucilage, carbon source, 775

- putrescine, uptake inhibits bacterial root colonization, 1096

Rhizostimulation, hydrocarbon-degrading bacteria, plant-bacterium pair as tool, 1197

Rhodococcus fascians

- on plant aerial parts, colonization, 599

- on tobacco, cortical cell division, de nova, 189

Ricci, P., 326

Riccio, A., 250, 823

Rice

-blast, gene tagging with transposon impala, 308

-blast and sheath blight, cover photo, October

-Magnaporthe grisea, pathogenesis-related gene family, 877

-Pyricularia grisea on: defense-related genes, subtractive hybridization, $685 ; m y b$ gene, infection and cell death, 527

-transgenic, disease resistance and wheat puroindolines, 1255

- vascular defense, peroxidase in xylem, 1411
Ritsema, T., 599

Rivas, S., 867

Rivilla, R., 663

Rodermel, S. R., 1206

Rodríguez-Palenzuela, P., 386

Rohel, E. A., 156

Roine, E., 394

Romantschuk, M., 394, 962

Romeis, T., 867

Ronald, P., 204

Roncero, M. I. G., 653

Rosenberg, C., 693

Rosenstein, Y., 1267

Ross, K., 31

Rossier, O., 629

Rosso, M.-N., 72

Rubio, M. C., 1178, 1189

Ryals, J., 1114

Saarilahti, H. T., 962

Sáenz, P., 278, 1032

Saitoh, H., 111

Sánchez, F., 1267

Sánchez-Navarro, J. A., 1051

San Francisco, M. J. D., 816

Sanjuan, J., 811

Sansavini, S., 508

San Segundo, B., 1327

Santos, R., 86, 758

Sauvage, C., 758

Savagnac, A., 678

Sawayanagi, T., 1043

Schäfer, W., 116

Schäfer-Pregl, R., 1420

Schoelz, J. E., 31

Scholthof, K.-B. G., 21

Scholz-Schroeder, B. K., 336, 1426

Schoonbeek, H., 562

Schornack, S., 629

Scott-Craig, J. S., 1436

Segers, G., 367

Semblat, J.-P., 72

Seo, S., 261

Sesbania rostrata, symbiosis disabled, lipopolysaccharide effect, 857

Seto, H., 261

Sevilla, M., 358

Shan, L., 1453

Shen, Y., 204

Sherwood, J. E., 1255

Shoemaker, R., 42

Siddique, A.-B. M., 173

Sigaud, S., 86

Silva, F. G., 204

Simidijeva, J., 609

Simmons, C. R., 947

Sinorhizobium meliloti

- on alfalfa, symbiosis and oxidative burst, 86

- fixNOQP expression, purine-related metabolite, 572

- ilvC mutants, on alfalfa, symbiosis, 55

—on Medicago truncatula, nematode role, 1168

- pyruvate dehydrogenase activity and nitrogen fixation, gene disruption, 811

$-r k p-3$ gene region, strain specificity and $\mathrm{K}$ antigen structure, 1395

- symbiotic efficiency, $n t r R$ gene mutation, 887 
Siomski, R., 1384

Slater, H., 768

Smant, G., 63

Smith, D. L., 1404

Snoeck, C., 678

Snoeijers. S. S., 316

Soberón, M., 572

Solanum spp. (see also Potato): speciation and Pto kinase family, diversification, 996

Son, O., 1286

Song, C. E., 80

Song, E.-S., 1286

Song, F., 1458

Sonti, R. V., 1335

Soto, M. J., 811

Soule, J. D., 1426

Soybean, cyst nematode

- cDNA peptide selection, clone, 536

- gene mapping, 42

- parasitic genes in esophageal glands, 1247

- screening and gene duplication, 63

Soybean mosaic virus

- hypersensitivity, Rsv1-mediated, 587

- N graft inoculation, hypersensitivity, cover photo, May

Spaink, H. P., 839

Spinosa, M. R., 823

Spiroplasma citri, on Catharanthus roseus, gene regulation, 225

Stacey, G., 1286

Stanley, J., 1125

Staskawicz, B. J., 181, 451

Stephens, S., 1395

Stiller, J., 422

Stolbur phytoplasma, on Catharanthus roseus, gene regulation, 225

Stone, P. J., 93

$\mathrm{Su}, \mathrm{H} ., 1206$

Sugarcane, Acetobacter diazotrophicus, growth effect and nitrogen incorporation, 358

Sugie, K., 725

Suillus bovinus, cytoskeleton, $\mathrm{Cdc} 42$ and actin relation, 135

Sullivan, T. A., 90

Sundaram, S., 618

Sutherland, P. W., 939

Suzuki, H., 618

Suzuki, K., 111

Sweat, T., 1411

Swinnen, E., 918

Syller, J., 1420

Tada, Y., 477

Taira, S., 394, 962

Takano, Y., 1149

Takata, Y., 477

Talbot, N. J., 1368

Tamai, A., 126, 1158

Tamarillo mosaic virus, infection and dark green islands, posttranscriptional gene silencing, 939

Tang, X., 1453

Tapesia yallundae, on wheat, ornithine decarboxylase, plaque formation, virulence, 1303

Tapio Palva, E., 931

Tarendeau, F., 225

Tarkka, M. T., 135

Tartarini, S., 508
Taté, R., 250, 823

Teplitski, M., 775

Terauchi, R., 111

Testa, A., 1444

Thomas, J., 1168

Thomas-Oates, J. E., 969, 1006, 1096

Tieman, D. M., 487

Tien, P., 1125

Tikhonovich, I. A., 471

Tobacco (see also Nicotiana spp.)

-black shank, elicitin gene expression, 326

-endoplasmic reticulum retrieval-retention signal, in tobacco leaves, agroinfiltration, cover photo, March

- plant transformation, Potato virus $X$, hypersensitivity, 1274

-Rhodococcus fascians on, cortical cell division, 189

Tobacco mosaic virus

—dysfunctional movement protein, microtubules, 895

-hypersensitivity, chimeric helicase sequences, 1086

-mutants, temperature-sensitive protein, heat shock, 914

—salicylic acid, signaling pathway, nitric oxide, 1458

-on tobacco, hypersensitivity and jasmonic acid, 261

-on transgenic tobacco, specific resistance, 1274

Tobias, C. M., 451

Tomato

-Cladosporium fulvum: avirulence gene Avr9 and nitrogen response, 316; resistance and avirulence gene products, binding evidence, 867 ; resistance and gene clusters, 508

-Clavibacter michiganensis, transposon mutagenesis system, 1312

- disease resistance, Pti5 gene, Pseudomonas syringae, 1453

- ethylene receptor gene, sensitivity and Xanthomonas campestris, 487

- foot and root rot, biocontrol with Pseudomonas chlororaphis, role of $p h z H$ gene, 1006

-hypersensitivity and Pseudomonas syringae, hrp genes, 234

-NADPH oxidase activity and oxidative burst in protoplasts, Arabidopsis domain protein, 1261

-Potato spindle tuber viroid, RNA silencing, 1332

-Pseudomonas syringae, wound response and coronatine, 214

Tomato mosaic virus, DNA infection system, plasmodesmata, 126

Tomato spotted wilt virus, on tomato and tobacco, maps and resistance, 242

Tomato yellow curl virus, gene $C 2$ effect, encodes pathogenicity determinant, 1125

Tosa, Y., 477

Touati, D., 86, 758

Toxin

-Alternaria alternata f. sp. lycopersici, infection role, 460

- phaseolotoxin, biosynthesis in

Pseudomonas syringae, 545

Transposon, impala, for gene tagging in rice blast, 308

Tsuchizaki, T., 1043

Tsuda, N., 725

Tsuge, T., 580

Tsyganov, V. E., 471

Tudzynski, B., 1293

Tudzynski, P., 1293

Tuinman, S., 969

Udvardi, M. K., 6

Ugaki, M., 1043

Uromyces fabae, on Vicia faba, biotrophic interaction, regulation of genes, 1319

Vaghchhipawala, Z., 42

Vähämiko, S., 962

Valent, B., 671

Valmeekam, V., 816

van den Ackereken, G., 629

van den Berg-Velthuis, G., 1444

van den Boogaart, T., 196

VandenBosch, K. A., 1364

van den Broek, D., 969

Van den Hooven, H. W., 867

van der Drift, K. M. G. M., 969

Van der Hoorn, R. A. L., 412, 867

van der Laak, J., 895

van der Lee, T., 1444

Vanderleyden, J., 426, 678, 918

Van der Ploeg, A., 412

Van Der Schueren, E., 288

Van der Weerden, G. M., 460

van Dooijeweert, W., 996

Van Monontagu, M., 288

van Spronsen, P. C., 839

van 't Clooster, J., 1444

van Wezel, R., 1125

Venard, P., 326

Venturia inaequalis, on apple, receptor-like genes, resistance, 508

Vercauteren, I., 288

Vernoud, V., 737

Verreth, C., 918

Vervoort, J., 867

Vicia faba, rust, biotrophic interaction, gene expression, 1319

Vila, L., 1327

Villalba, F., 308

Vinatzer, B. A., 508

Virtaharju, O., 962

Vishnupriya, M. R., 1335

Vlassak, K., 918

Vleeshouwers, V. G. A. A., 996

Voegele, R. T., 1319

Vojnov, A. A., 768

von Roepenack-Lahaye, E., 785

Voroshilova, V. A., 471

Walling, L. L., 214

Walton, J. D., 1436

Wang, X. (N.C.), 536

Wang, X.-J. (China), 1261

Warren, R. F., 1453

Webb, K. J., 1178

Wehrfritz, J.-M., 867

Wen, F., 196

Wheat

- Mycosphaerella graminicola, biotrophic status, 156

- $\tan$ spot, ToxB gene characterization, 675 
-Tapesia yallundae, infection plaque formation, ornithine decarboxylase, 1303

- transgenic, genes mediating fungal resistance, 832

Wiegand, C., 914

Willard, L. H., 1411

Willtis, M., 1114

Wilson, R. A., 980

Wirsel, S. G. R., 1319

Wise, R. P., 1376

Woloshuk, C. P., 955

Wolucka, B., 857

Wood, M., 1016

Wubben, M. J. E., II, 1206

Xanthomonas spp.

-X. campestris: on crucifers, xanthan

biosynthesis, regulation, gum operon, 768;

on pepper, hydroxycinnamoyl-tyramine

conjugate induction, 785; on pepper and tomato, AvrBs4 protein, recognition and gene mapping, 629; on tomato, hypersensitivity, ethylene receptor gene effect, 487

$-X$. oryzae: flagellar operon region, $f l h F$ characterization, 204; on rice, extracellular polysaccharide production and virulence, genomic locus, 1335; on rice, vascular defense, peroxidase in xylem, 1411

Xing, T., 1261

Xiong, L., 685

Xylella fastidiosa, transposon mutagenesis, Tn5 synaptic complexes, 701

Yakoby, N., 988

Yalpani, N., 947

Yamakawa, H., 261

Yamamoto, H., 749

Yamamoto, M., 749

Yamamura, S., 111

Yan, Y., 63
Yang, J. M., 793

Yang, S.-J., 804

Yang, Y., 527, 685

Yashitola, J., 1335

Yoshioka, H., 725

Young, S. A., 1411

Yu, G.-X., 1376

Yuan, J., 234

Zellermann, E.-M., 1312

Zhang, H. (Canada), 1404

Zhang, H.-B. (Texas), 508

Zhang, L., 1063

Zhang, Y., 1131

Zhao, T., 1453

Zheng, H., 1063

Zhou, J.-M., 1453

Zhu, L., 1453

Zucchero, J. C., 1463

Zuck, M., 431 